\section{Efficient and improved edge detection via a hysteresis thresholding method}

\author{
Sajid Khan ${ }^{1, *}$, Dong-Ho Lee ${ }^{2}$, \\ Muhammad Asif Khan ${ }^{3}$, Abdul Rehman Gilal ${ }^{4}$, \\ Junaid Iqbal $^{5}$ and Ahmad Waqas ${ }^{4}$
}

${ }^{1}$ Center of Excellence for Robotics, Artificial Intelligence and Blockchain, Sukkur IBA University, Sukkur, Pakistan

${ }^{2}$ School of Electrical Engineering,

Hanyang University ERICA Campus, Ansan, South Korea

${ }^{3}$ Department of Electrical Engineering, and

${ }^{4}$ Department of Computer Science, Sukkur IBA University,

Sukkur, Pakistan

${ }^{5}$ Department of Mechatronics Engineering,

Hanyang University ERICA Campus, Ansan, South Korea

\begin{abstract}
Hysteresis thresholding is a popular technique for automatic edge detection. However, calculating reasonably high and low thresholds using an unsupervized method remains an issue. Conventional low and high threshold-linking methods sometimes produce noisy edges and fail to detect some obvious edges. Here, a novel edge detection algorithm is proposed that provides efficient calculation of thresholds, and links edge maps for extraction of the final edge map at low complexity. The proposed method suppresses unwanted noisy edges while efficiently preserving obvious edges. The simulation results show that the proposed method provides better results in terms of performance and computation time. Thus, it can be applied to any feature image analysed by an edge detector.
\end{abstract}

Keywords: Edge detection, hysteresis method, low and high thresholds, noise suppression.

EDGE detection provides a meaningful description of object boundaries from the scenes of images ${ }^{1-3}$ and requires applying automatic thresholding to the gradients of an image. There are two categories of thresholding, dynamic thresholding ${ }^{4,5}$ and static thresholding ${ }^{1,4,6}$. Dynamic thresholding is a complex process that requires calculation of different thresholds for various regions of the image. Static thresholding falls into two sub-categories: single thresholding ${ }^{7,8}$ and hysteresis thresholding ${ }^{9-13}$. Single thresholding applies single calculated threshold on the gradient map. Unlike single thresholding, hysteresis thresholding is based on determining low and high thresholds followed by an edge linking process to detect edges.

In single thresholding, if the calculated threshold is low, it will result in the detection of false/noisy edges ${ }^{7,8}$. On the other hand, if the threshold selected is very high, it will result in ignoring many important true edges.

\footnotetext{
*For correspondence. (e-mail: sajidkhan@iba-suk.edu.pk)
}

Hysteresis thresholding calculates both high and low thresholds and uses them to produce a final edge map. It provides far better results than single thresholding if the low and high thresholds are reasonably calculated and their thresholded images are processed properly.

Guha and Saha ${ }^{10}$ proposed a method that detects edges after estimation of blur scale of a region. Canny ${ }^{9}$ proposed hysteresis thresholding-based edge detection, whereas Rong et al. ${ }^{13}$ proposed an algorithm based on Canny's method, but with modification. For example, they have replaced the Gaussian filter of Canny ${ }^{9}$ with an adaptive filter and non-maximal suppression-based edge thinning with morphological thinning ${ }^{13}$.

A major contribution was made by Medina-Carnicer et $a l .^{11,12}$ to automatically calculate hysteresis thresholds. They used all $L$ bins of the histogram as candidates and processed them using the receiver operating characteristic curve to evaluate the low and high thresholds. They followed previous work by selecting a range of histogram bins instead of all $L$ bins $^{12}$. This requires initial calculation of the Rosin ${ }^{7}$ and Otsu ${ }^{8}$ thresholds to define the candidates of the threshold.

The problem with conventional unsupervised hysteresis thresholding methods is that they fail to calculate a reasonable pair of low and high thresholds. The calculated high threshold edge map of Carnicer et al. ${ }^{11}$ misrepresents some obvious edges. On the other hand, the calculated low threshold of Medina-Carnicer et al. ${ }^{12}$ does not contain all edge pixels of obvious edges.

This study proposes a low-complexity hysteresis thresholding method with improved calculation of low and high threshold pair. The objective is to provide a simple and efficient way to reduce the number of threshold candidates and maintain a proper gap between the calculated low and high thresholds. A noise suppression-based edge map-linking method is also proposed.

Let us discuss conventional threshold calculation methods. The notation $I$ is used for the input image and $G$ is used for describing the gradient image obtained using a specific gradient operator. The histogram $H$ is a gradient histogram that contains $L$ bins. Every histogram bin has a certain gradient intensity value $\left\{T_{0}, T_{1}, T_{2}, \ldots, T_{L-1}\right\}$ that can be used as a threshold candidate. Each gradient intensity value corresponds to its normalized probability value, $\left\{\rho_{T_{0}}, \rho_{T_{1}}, \rho_{T_{2}}, \ldots, \rho_{T_{L}}\right\}$. The low and high thresholds are denoted by $T_{\text {low }}$ and $T_{\text {high }}$ respectively.

All the methods use a gradient image to select a single threshold or two. Conventions of TP, TN, FP and FN are used for true positives, true negatives, false positives and false negatives respectively. The equations for calculating these are similar for all methods.

Medina-Carnicer et $a l^{12}$ used the Otsu threshold ( $\left.T_{\text {Otsu }}\right)$ as the subset and Rosin threshold $\left(T_{\text {Rosin }}\right)$ as the superset of the thresholds to be calculated. If the defined set turns out to be an empty set, it uses all $L$ bins of the histogram as candidates. After selecting a set of candidates, it 
calculates the low and high thresholds using the following equation

$$
\left(T_{\text {low }}, T_{\text {high }}\right)={ }_{t_{\text {low }}, t_{\text {high }}}^{\arg \max } \mu\left(G_{t_{\text {low }}, t_{\text {high }}}(I)\right),
$$

where

$$
\begin{aligned}
& \mu\left(G_{t_{\text {low }}, t_{\text {high }}}(I)\right)=\mu_{1}\left(G_{\mathrm{Otsu}}\right)+\mu_{1}\left(G_{\mathrm{Rosin}}\right), \\
& \mu_{1}\left(G_{X}\right)=X_{G_{t_{\text {low }}, t_{\text {high }}}^{2}}(I), G_{X}(I), \\
& X_{C}^{2}=\frac{\widehat{\operatorname{Sn}}_{C}-Q_{C}}{1-Q_{C}} \frac{\widehat{\operatorname{Sp}}_{C}-\left(1-Q_{C}\right)}{Q_{C}}, \\
& C=G_{t_{\text {low }}, t_{\text {high }}}(I), G_{X}(I), \\
& Q_{C}=\operatorname{TP}(C)+\operatorname{FP}(C), \\
& \widehat{\operatorname{Sn}}_{C}=\frac{\operatorname{TP}(C)}{\operatorname{TP}(C)+\operatorname{FN}(C)}, \\
& \widehat{\operatorname{Sp}}_{C}=\frac{\operatorname{TN}(C)}{\operatorname{FP}(C)+\operatorname{TN}(C)} .
\end{aligned}
$$

Here, $\mathrm{TP}(C), \mathrm{TN}(C), \mathrm{FP}(C)$ and $\mathrm{FN}(C)$ are the true positives, true negatives, false positives and false negatives of $G_{t_{\text {low }}, t_{\text {high }}}(I)$ when comparing with edge map $G_{\mathrm{X}}(I)$. $G_{t_{\text {low }}, t_{\text {high }}}(I)$ represents edge maps that are based on all possible combinations of low and high thresholds.

In many cases, Otsu provides a threshold that is smaller than the Rosin threshold, such that Medina-Carnicer et $a l .{ }^{12}$ keep switching to $L$ candidates. Also, $G_{t_{\text {low }}, t_{\text {high }}}(I)$ is an edge map that results from the application of all possible pairs of $\left(t_{\text {low }}, t_{\text {high }}\right)$ from the set of candidates. These factors result in the high complexity of the method proposed by Medina-Carnicer et al. ${ }^{12}$.

Rosin is a single thresholded method that uses the histogram of the gradient image. It processes a straight line that connects the peak of the histogram to its high end. The bin of the histogram that provides the maximum perpendicular distance from the straight line is selected as the threshold. Rosin thresholding provides the low threshold value if the peak is close to the origin, and vice versa. If the histogram of $G$ is negatively skewed, the Rosin method will select a threshold that results in the detection of few edges. Also, it is a single thresholding method that detects edges in the presence of some isolated noisy edges.

Medina-Carnicer et al. ${ }^{11}$ proposed an efficient hysteresis thresholding method that uses all $L$ bins of the histogram as candidates for thresholds. The method selects every bin of the histogram $H$ as the reference threshold. It then compares them with all $L$ bins of $H$ to calculate TP, FP, TN and FN. The calculated parameters are processed to find $T_{\text {low }}$ using the following equation

$$
T_{\text {low }}=\frac{(L-2) P}{1+2 P},
$$

Here, $P=\overline{\mathrm{TP}}+\overline{\mathrm{FP}}$ is the average number of positive detections of the candidate $x$, and $T_{\text {high }}$ is the threshold that corresponds to the following condition

$$
\widehat{\mathrm{FP}}(x)=\widehat{\mathrm{FN}}(x)
$$

Medina-Carnicer et al. $^{11}$ do not provide any criteria for candidate selection and use all $L$ bins of the histogram. In most cases, their $T_{\text {high }}$ is much larger than $T_{\text {low }}$ (ref. 11). Large gap between thresholds results in missing obvious edges in connecting low and high threshold edge maps.

In hysteresis thresholding, it is necessary for the high threshold edge map to represent all the obvious edges, whether they are completely or partially detected. If the calculated high threshold is large, it will misrepresent an obvious edge in the high threshold edge map. Misrepresentation of true edges will result in missing obvious edges by linking them to their corresponding low threshold edges. Also, the final image, which is the result of connecting low and high threshold images, should not contain noisy edges.

The proposed method calculates low and high thresholds with reasonable gap and connects them using the noise suppression-based connectivity method. It ensures preservation of all obvious edges and suppression of noisy ones from the low threshold edge map. Unlike Medina-Carnicer et al. ${ }^{11,12}$, the proposed candidate selection criteria limit the number of candidates to a great extent. It does not require any kind of switching to all $L$ candidates, since there is no chance of failure of the candidate reduction method. The proposed method is divided into the following steps: (1) histogram threshold-based screening, (2) threshold calculation, and (3) linking low and high edge maps. All the involved steps are shown in Figure 1 and explained in the following.

Histogram threshold-based screening: The histogram of the gradient image $G$ contains $L$ bins $\left\{T_{0}, T_{1}, T_{2}, \ldots\right.$, $\left.T_{L-1}\right\}$ with normalized probability $\left\{\rho_{T_{0}}, \rho_{T_{1}}, \rho_{T_{2}}, \ldots, \rho_{T_{L-1}}\right\}$. of occurrence. In selecting candidates for threshold selection, bins with zero probability of occurrence are totally neglected. The number of candidates is further reduced to obtain a final set of candidates. This final set can be defined using the following equation

$$
X=\left\{x \mid x \in T_{k} \text { as } k=0,1, \ldots, N_{X}-1, \rho_{x}>\delta\right\},
$$

where $\delta$ is the histogram threshold calculated as shown below 


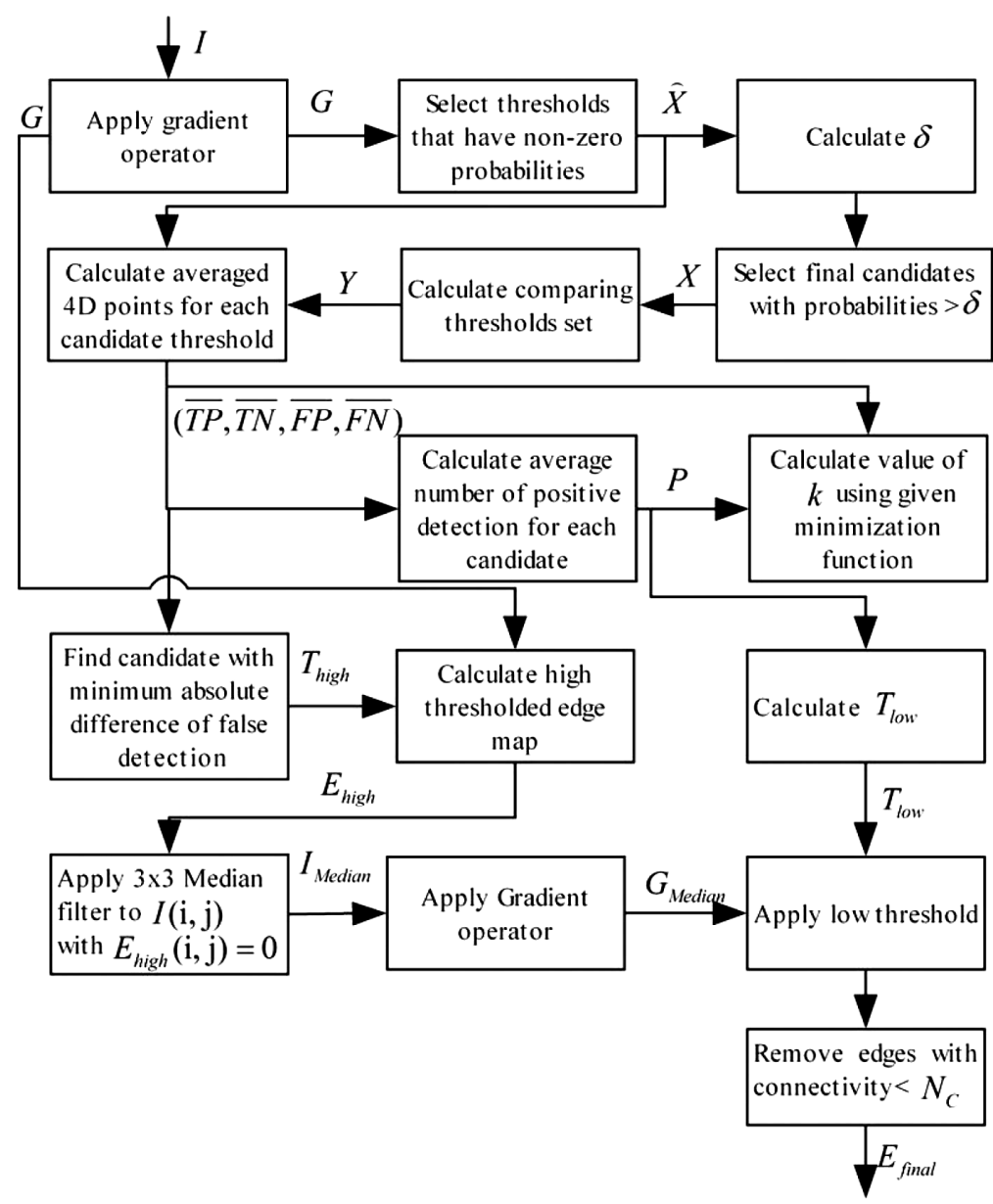

Figure 1. Flowchart of the proposed edge detection method.

$$
\delta=\frac{\rho_{\hat{x}}(1: n)}{n}
$$

Here, $\hat{X}$ is a set having $N_{\hat{X}}$ elements and $n=1 N_{\hat{X}} * \alpha$, and $|$.$| is rounded-off to the closest integer value. The set$ $\hat{X}$ is defined as

$$
\hat{X}=\left\{\hat{x} \mid \hat{x} \in T_{c} \text { as } c=0,1, \ldots, N_{\hat{X}}-1, \rho_{\hat{x}}>0 \text { and } \rho_{T_{c}} \geq \rho_{T_{c-1}}\right\},
$$

where $\alpha$ is a parameter that is proportional to the number of candidates selected. $\alpha$ also helps in maintaining a proper gap between the low and high thresholds.

Threshold calculation: Let $G_{A}$ be an edge map that is obtained due to the application of threshold $T_{A}$ on the gradient image $G$. In order to calculate TP, TN, FP and FN for $G_{A}$, threshold $T_{\text {Ref }}$ is applied on $G$ to obtain a reference edge map $G_{\text {Ref. }}$. There are two conditions that can be used to calculate the 4D points (TP, TN, FP, FN).

If $T_{\mathrm{Ref}}>T_{A}$,

$$
\operatorname{TP}\left(T_{A}, T_{\mathrm{Ref}}\right)=\sum_{0}^{N_{\mathrm{Ref}}} \rho_{T_{N_{\mathrm{Ref}}}},
$$

Here, $N_{\text {Ref }}$ and $N_{A}$ are the indices of the reference set $X$ and comparing set $Y$ respectively. The threshold $T_{\text {Ref }}$ is a member of set $X$ and threshold $T_{A}$ is from set $Y$. 
Figure 2 shows the TP, TN, FP and FN results for both cases.

Set $Y$ can be expressed as follows

$$
Y=\left\{y \mid y \in T_{r} \text { as } r=0,1, \ldots, N_{Y}-1, \rho_{\mathrm{y}}>\delta \text { and } y \neq x\right\} \text {. }
$$

Let $G_{x}$ be a reference binary edge map and $G_{y}$ be the binary edge map to be compared. The above equations can be used to obtain $N_{Y}$ 4D points (TP $(y, x), \operatorname{TN}(y, x)$, $\mathrm{FP}(y, x), \mathrm{FN}(y, x))$ for each candidate $x . \quad N_{Y}=N_{X}-1$ denotes the length of set $Y$. All $N_{Y}$ 4D points were averaged and used to perform ROC analysis as follows

$$
\begin{aligned}
& \overline{\mathrm{TP}}_{x}=\frac{1}{N_{Y}} \sum_{r=0, y_{r} \neq x}^{N_{Y}-1} \mathrm{TP}\left(y_{r}, x\right), \\
& \overline{\mathrm{TN}}_{x}=\frac{1}{N_{Y}} \sum_{r=0, y_{r} \neq x}^{N_{Y}-1} \mathrm{TN}\left(y_{r}, x\right), \\
& \overline{\mathrm{FP}}_{x}=\frac{1}{N_{Y}} \sum_{r=0, y_{r} \neq x}^{N_{Y}-1} \mathrm{FP}\left(y_{r}, x\right), \\
& \overline{\mathrm{FN}}_{x}=\frac{1}{N_{Y}} \sum_{r=0, y_{r} \neq x}^{N_{Y}-1} \mathrm{FN}\left(y_{r}, x\right),
\end{aligned}
$$

Here, $y_{r}$ denotes the $r$ th element of set $y$. The low threshold $T_{\text {low }}$ can be calculated as follows

$$
T_{\text {low }}=P_{K} * x^{\max }
$$

where $x^{\max }$ is the maximum gradient intensity of the candidate set $X . P_{K}=\overline{\mathrm{TP}}_{x_{K}}+\overline{\mathrm{FP}}_{x_{K}}$ is the average number of positive detections for a candidate at index $K$ of the candidate set $x$. Index $K$ is calculated using the equation given below.

$$
K=\min _{s \in\left\{0,1, \ldots, N_{X-1}\right\}} \sqrt{\left(1-\frac{\overline{\mathrm{TP}}_{x_{S}}}{P_{S}}\right)^{2}+\left(1-\frac{\overline{\mathrm{TN}}_{x_{S}}}{1-P_{S}}\right)^{2}},
$$

where $N_{X}$ refers to the length of the candidate set $X$. The high threshold $T_{\text {high }}$ can be calculated using the following equations

$$
\begin{aligned}
& T_{\text {high }}=x_{M}, \\
& M=\min _{l \in\left\{0,1, \ldots, N_{X-1}\right\}}\left|\overline{\mathrm{FP}}_{x_{l}}-\overline{\mathrm{FN}}_{x_{l}}\right|,
\end{aligned}
$$

where $\overline{\mathrm{FP}}_{x_{l}}$ and $\overline{\mathrm{FN}}_{x_{l}}$ refers to the averaged false detections for the $l$ th threshold from set $X$.
Linking low and high edge maps: Most conventional methods link all edges of the high threshold edge map with those of the low threshold edge map. Such techniques result in detection of noisy edges. The proposed method preserves all the edges of the high threshold edge map by applying noise suppression to the rest of the edges. The steps for applying hysteresis thresholds are given as: (i) Obtain a high threshold edge map using $T_{\text {high }}$ (ii) Apply $3 \times 3$ median filter to all input intensity pixels, except the detected high threshold edges. (iii) Calculate gradient image of filtered image and apply low threshold to it. (iv) Remove edges that have a number of connected edge pixels less than $N_{c}$.

For simulation, gradient operators such as simple differential operator, Prewitt, and Sobel were applied to analyse different cases. The simple differential operator is highly prone to noise and makes a good case to analyse the performance of an edge detector. The values of $\alpha=0.7$ and $N_{c}=25$ were used on the basis of extensive simulations.

The proposed algorithm was compared to the MedinaCarnicer et al. ${ }^{11,12}$ and Rosin ${ }^{13}$ for subjective and objective evaluation. Figure 3 shows the images that were used to produce the results.

Figure 4 shows the results of applying the conventional and proposed methods to banana and Lena images. The former image has minor image intensity variations in the region of the banana that results in weak gradient values along edges. Weak gradient makes an edge difficult to

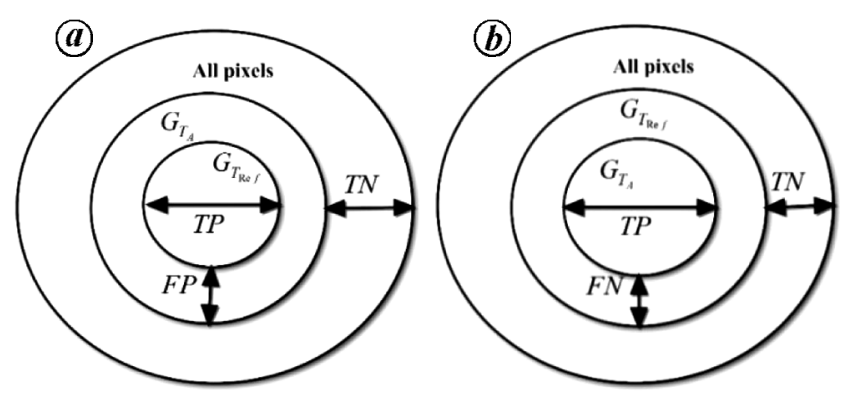

Figure 2. Graphical illustration of TP, TN, FP and FN when (a) $T_{\mathrm{Ref}}>T_{A}$ and $(\boldsymbol{b}) T_{\mathrm{Ref}}<T_{A}$.

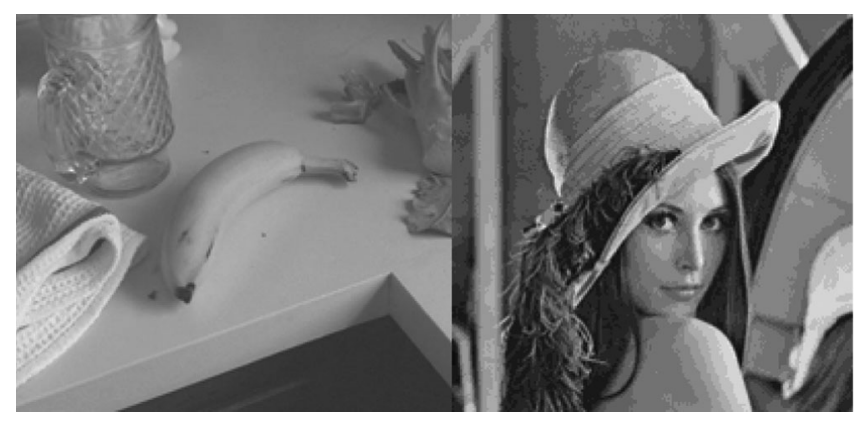

Figure 3. Test images used as input for subjective evaluation. 

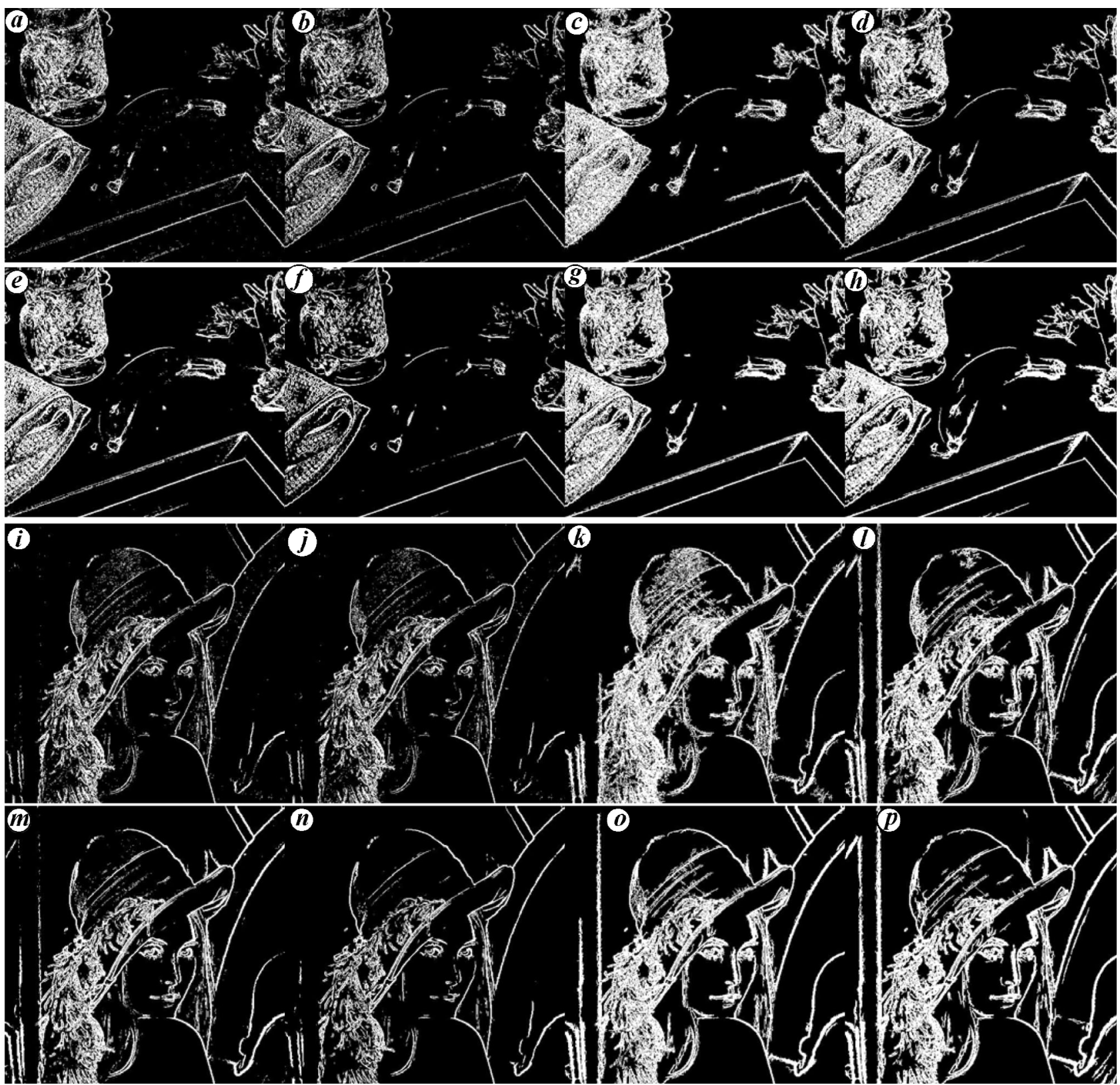

Figure 4. Results obtained on applying algorithms using simple differential and Prewitt gradient operators, on banana and Lena images respectively: $\boldsymbol{a}, \boldsymbol{e}, \boldsymbol{i}, \boldsymbol{m}, \mathrm{Rosin} ; \boldsymbol{b}, \boldsymbol{f}, \boldsymbol{j}, \boldsymbol{n}$, Medina-Carnicer et al. ${ }^{12} ; \boldsymbol{c}, \boldsymbol{g}, \boldsymbol{k}, \boldsymbol{o}$, Medina-Carnicer et al. ${ }^{11} ; \boldsymbol{d}, \boldsymbol{h}, \boldsymbol{l}, \boldsymbol{p}$, proposed method.

Table 1. Number of candidates selected after applying histogram threshold screening

\begin{tabular}{lclc}
\hline Image & No. of candidates & Image & No. of candidates \\
\hline Baboon & 44 & Feather & 48 \\
Banana & 28 & Lax & 49 \\
Basket & 40 & Lena & 47 \\
Briefcase & 55 & Milkdrop & 59 \\
Egg & 30 & Pepper & 55 \\
\hline
\end{tabular}

detect. Lena image contains a variety of low-contrast and high-contrast edges. Both images are good cases for subjective evaluation of the algorithms. Figure $4 a-d$, $i-l$ shows results of application of the conventional and proposed methods on gradient images obtained due to simple differential operator. Figure $4 e-h, m-p$ shows application of the algorithms on gradient image obtained after application of the Prewitt operator. The simple differential operator takes difference along $x$-axis and $y$-axis to produce $x$-directional and $y$-directional gradient. This operator is rarely used as it is highly prone to noise. On the other hand, Prewitt is a widely used operator.

Isolated noise can be seen in all the results, especially for the case of simple differential operator since Rosin is a single threshold method. Medina-Carnicer et al. ${ }^{9,10}$ failed to detect some of the obvious edges. They also 


\section{RESEARCH COMMUNICATIONS}
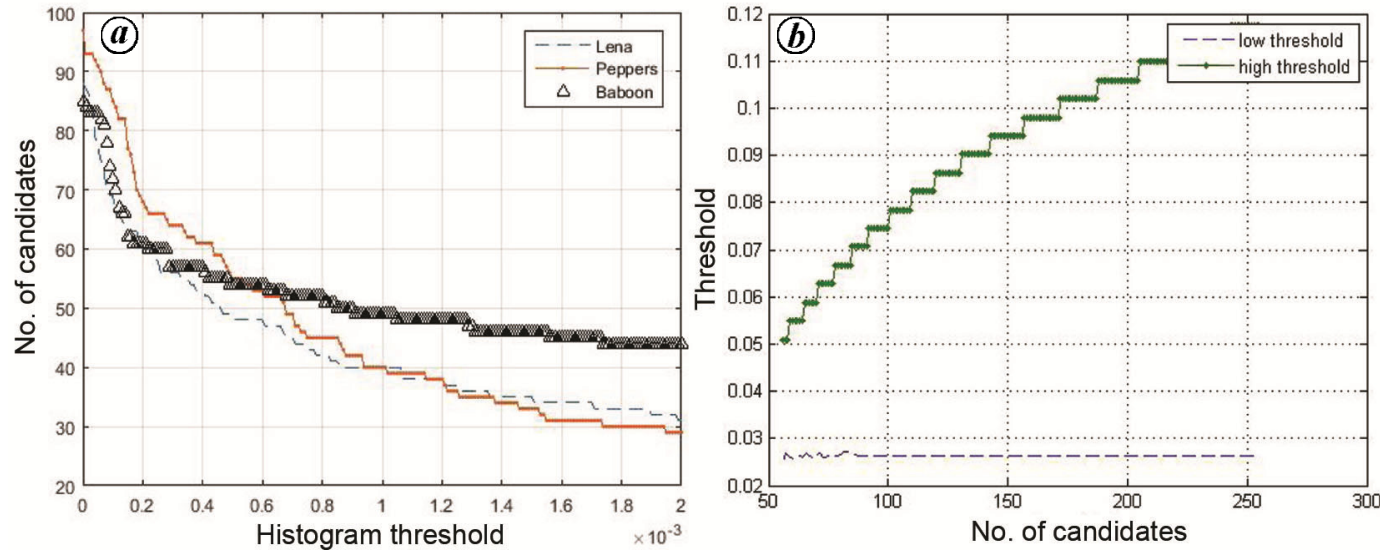

Figure 5. Analysis of variation of one parameter with respect to another. $\boldsymbol{a}$, Number of candidates selected for specific histogram thresholds. $\boldsymbol{b}$, The calculated low and high thresholds for different number of candidates.

Table 2. Comparison of calculated thresholds for different images

\begin{tabular}{|c|c|c|c|c|c|c|c|}
\hline \multirow[b]{2}{*}{ Image } & \multicolumn{2}{|c|}{ Medina-Carnicer et $_{\text {al. }}{ }^{12}$} & \multicolumn{2}{|c|}{ Medina-Carnicer et al. ${ }^{11}$} & \multicolumn{2}{|c|}{ Proposed method } & \multirow{2}{*}{$\frac{\text { Rosin }}{T_{\text {Rosin }}}$} \\
\hline & $T_{\text {low }}$ & $T_{\text {high }}$ & $T_{\text {low }}$ & $T_{\text {high }}$ & $T_{\text {low }}$ & $T_{\text {high }}$ & \\
\hline Baboon & 0.0980 & 0.1020 & 0.0501 & 0.1333 & 0.0468 & 0.0627 & 0.1020 \\
\hline Banana & 0.0313 & 0.0353 & 0.0151 & 0.0745 & 0.0143 & 0.0235 & 0.0196 \\
\hline Basket & 0.1098 & 0.1137 & 0.0500 & 0.1216 & 0.0469 & 0.0588 & 0.1137 \\
\hline Briefcase & 0.0784 & 0.0824 & 0.0285 & 0.1451 & 0.0259 & 0.0510 & 0.0510 \\
\hline Egg & 0.0392 & 0.0431 & 0.0173 & 0.0902 & 0.0163 & 0.0235 & 0.0274 \\
\hline Feather & 0.0823 & 0.0863 & 0.0405 & 0.1176 & 0.0388 & 0.0549 & 0.0863 \\
\hline Lax & 0.0745 & 0.0784 & 0.0437 & 0.1490 & 0.0394 & 0.0588 & 0.0627 \\
\hline Lena & 0.0588 & 0.0627 & 0.0262 & 0.1176 & 0.0250 & 0.0431 & 0.0431 \\
\hline Milkdrop & 0.0824 & 0.0863 & 0.0189 & 0.1333 & 0.0190 & 0.0314 & 0.0392 \\
\hline Pepper & 0.0784 & 0.0824 & 0.0258 & 0.1333 & 0.0255 & 0.0431 & 0.0431 \\
\hline
\end{tabular}

Table 3. Statistical measures obtained by applying edge detectors to the images used

\begin{tabular}{|c|c|c|c|c|}
\hline & Rosin & $\begin{array}{c}\text { Medina- } \\
\text { Carnicer et al. }{ }^{12}\end{array}$ & $\begin{array}{c}\text { Medina- } \\
\text { Carnicer et al. }\end{array}$ & Proposec \\
\hline IMP & 0.5563 & 0.7557 & 0.4221 & 0.7905 \\
\hline FP & 2809.5 & 866.75 & 4365.5 & 1058.25 \\
\hline $\mathrm{FN}$ & 609.75 & 864.25 & 417.5 & 370.75 \\
\hline $\mathrm{TP}$ & 2433.75 & 2179.25 & 2626 & 2672.75 \\
\hline$P_{\text {nd }}$ & 0.1401 & 0.2356 & 0.0601 & 0.0912 \\
\hline$P_{\text {co }}$ & 0.4864 & 0.6679 & 0.3962 & 0.7296 \\
\hline$P_{\mathrm{fa}}$ & 0.5136 & 0.23 & 0.604 & 0.2703 \\
\hline$d_{\mathcal{L} 2}^{4}$ & 0.8775 & 0.559 & 1.034 & 0.453 \\
\hline
\end{tabular}

detected some noisy edges using a simple differential operator. For Prewitt operator, Medina-Carnicer et al. ${ }^{9,10}$ missed obvious edges (Figure 4). The proposed method, on the other hand, outperformed all the other methods by detecting all the important edges and also suppressed noise completely.

Table 1 shows the number of candidates selected for some images when histogram threshold-based screening was applied. It is clear from the table that probability- based screening helps in selecting a small set of candidates, unlike the case of Medina-Carnicer et al. ${ }^{11,12}$ who used all $L$ bins of the histogram as candidate thresholds. Medina-Carnicer et al. $^{12}$ also switched back to using all $L$ bins as candidates when the Otsu threshold was smaller compared to the Rosin threshold.

Table 2 shows the thresholds calculated for 10 test images using the methods of Medina-Carnicer et al. ${ }^{11,12}$, the proposed method, and Rosin method. Prewitt operator was used for gradient calculation. It is clear from Table 2 that the method of Medina-Carnicer et al. ${ }^{11}$ produced too large a difference between the low and high thresholds. It missed some obvious edges because its high threshold edge map does not have representation of most of the true edges. Medina-Carnicer et al. ${ }^{11}$, also calculated low thresholds that were too far from the desired range of thresholds for most cases. Rosin being a single threshold method misses obvious edges and also produces false detection.

The performance of the proposed edge detector method was compared with conventional methods using parameters given by Gonzaga ${ }^{14}$. Parameters such as TP, FP, FN, and similarity between two contours defined using the 
figure of merit of Pratt (IMP) were calculated. Percentage of correct detection $\left(P_{\mathrm{co}}\right)$, percentage of missing $\left(P_{\mathrm{nd}}\right)$, percentage of false alarm $\left(P_{\mathrm{fa}}\right)$ were calculated on the basis of the calculated TP, FP and FN. Euclidean distance $\left(d_{\mathcal{L} 2}^{4}\right)$ to the point $P=\left(P_{\text {co }}\right.$, IMP, $\left.P_{\text {nd }}, P_{\text {fa }}\right)$ was also calculated. All the parameters were calculated for various images and averaged (Table 3). The dataset of FernándezGarcia et $a l .{ }^{15}$ was used for comparison, as it provides ground truths of edges. Table 3 shows that the proposed method provides better TP and FN compared to other methods, and the second best FP. The Euclidean distance $d_{\mathcal{L} 2}^{4}$ that should be 0 in the perfect case is smallest for the proposed method. The ideal value of point $P=\left(P_{\mathrm{co}}\right.$, IMP, $\left.P_{\text {nd }}, P_{\text {fa }}\right)$ is $(1,1,0,0)$. The proposed method shows the desired results for $P_{\text {co }}$ and IMP, whereas values of $P_{\text {nd }}$ and $P_{\mathrm{fa}}$ are the second best with a slight margin.

The parameter that controls the number of candidates used is the histogram threshold is denoted by $\delta$. It is inversely proportional to the parameter $\alpha$. Increasing $\delta$ decreases the number of candidates, and vice versa. Figure $5 a$ shows the number of candidates selected for different histogram thresholds.

A high value of $\delta$ results in a reduction of the complexity of the algorithm due to lower number of candidates. However, it results in the detection of noisy edges in the high threshold edge map because the difference $T_{\text {high }}-$ $T_{\text {low }}$ will become negligible. Due to a very small $T_{\text {high }}$ value, the high threshold edge map will contain noisy edges as well. Figure $5 b$ shows the low and high thresholds calculated for different number of candidates. Candidates were ignored on the basis of smaller probability in the histogram.

A small number of candidates leads to a smaller value of $T_{\text {high }}$ because the latter is calculated on the basis of minimization of $\left|\overline{\mathrm{FP}}_{x(t)}-\overline{\mathrm{FN}}_{x(t)}\right|$. It is observed that most of the small probability bins lie at the tail of the gradient histogram. Increasing the value of $\delta$ results in the selection of fewer candidates near the tail of the histogram. Therefore, for each $x$, the condition $x>y$ is relatively more frequently justified, because possible candidates of $T_{\text {high }}$ are far from the tail of the histogram. Frequent justification of $x>y$ results in the addition of some positive numbers to $\overline{\mathrm{FP}}_{x}$, according to eq. (16). In such a situation, nothing is added to $\overline{\mathrm{FN}}_{x}$ according to eq. (17). Hence, with decrease in the number of candidates, $\overline{\mathrm{FP}}_{x}$ increases. The gap between $\overline{\mathrm{FN}}_{x}$ and $\overline{\mathrm{FP}}_{x}$ continues to decrease and a minimum value of $\left|\overline{\mathrm{FP}}_{x(t)}-\overline{\mathrm{FN}}_{x(t)}\right|$ will be obtained at an early threshold.

Hysteresis thresholding provides efficient edge detection. However, automatic determination of the best threshold pair, instead of a single threshold, makes it difficult to use. This study proposes an efficient hysteresis threshold calculation method with efficient candidate selection criteria. The proposed algorithm reduces the gap between high and low thresholds that results in the detection of more true edges. The proposed edge linking method also ensures that all true edges are detected in the case of no or negligible noise.

1. Zhang, K., Zhang, Y., Wang, P., Tian, Y. and Yang, J., An improved Sobel edge algorithm and FPGA implementation. Procedia Comput. Sci., 2018, 131, 243-248.

2. Lee, D. H., A simple, high performance edge-adaptive deinterlacing algorithm with very low complexity. In IEEE International Conference on Consumer Electronics, Las Vegas, NV, USA, January 2012, pp. 636-637.

3. Khan, S. and Lee, D., Efficient deinterlacing method using simple edge slope tracing. Opt. Eng., 2015, 54, 103108-1-103108-10.

4. Bias, S. and Kale, I., Mobile hardware based implementation of a novel, efficient, fuzzy logic inspired edge detection technique for analysis of malaria infected microscopic thin blood images. Procedia Comput. Sci., 2018, 141, 374-381.

5. Hossain, F., Asaduzzaman, M., Yousuf, M. A. and Rahman, M. A., Dynamic thresholding based adaptive canny edge detection. Int. J. Comput. Appl., 2016, 975, 37-41.

6. Yang, L., Zhao, D., Wu, X., Li, H. and Zhai, J., An improved Prewitt algorithm for edge detection based on noised image. In International Congress on Image and Signal Processing, Shanghai, China, October 2011, vol. 3, pp. 1197-1200.

7. Rosin, P. L., Unimodal thresholding. Pattern Recogn., 2001, 34, 2083-2096.

8. Otsu, N., A threshold selection method from gray-level histograms. IEEE Trans. Syst., Man, Cybern., 1979, 9, 62-66.

9. Canny, J., A computational approach to edge detection. IEEE Trans. Pattern Anal. Mach. Intell., 1986, 6, 679-698.

10. Guha, I. and Saha, P. K., A new algorithm for local blur-scale computation and edge detection. In International Symposium on Visual Computing, Las Vegas, NV, USA, November 2018, pp. 598-606.

11. Medina-Carnicer, R., Madrid-Cuevas, F. J., Carmona-Poyato, A. and Muñoz-Salinas, R., On candidates selection for hysteresis thresholds in edge detection. Pattern Recogn., 2009, 42, 1284 1296.

12. Medina-Carnicer, R., Carmona-Poyato, A., Muñoz-Salinas, R. and Madrid-Cuevas, F. J., Determining hysteresis thresholds for edge detection by combining the advantages and disadvantages of thresholding methods. IEEE Trans. Image Process., 2009, 19, 165173.

13. Rong, W., Li, Z., Zhang, W. and Sun, L., An improved CANNY edge detection algorithm. In IEEE International Conference on Computer Science and Engineering, Qingdao, China, 1 August 2009, pp. 577-582.

14. Gonzaga, A., Method to evaluate the performance of edge detector. In International Conference on Intelligent Systems Design and Applications, Rio de Janeiro, Brazil, 2007, pp. 341-346.

15. Fernández-García, N. L., Carmona-Poyato, A., Medina-Carnicer, R. and Madrid-Cuevas, F. J., Automatic generation of consensus ground truth for the comparison of edge detection techniques. Image Vis. Comput., 2008, 26, 496-511.

Received 20 February 2019; revised accepted 20 November 2019

doi: $10.18520 / \mathrm{cs} / \mathrm{v} 118 / \mathrm{i} 6 / 954-960$ 